\title{
Avaliação técnica e econômica da extração de madeira com forwarder em diferentes volumes por árvore e comprimentos de toras
}

\author{
Sérgio Luis Martins dos Santos ${ }^{1}$ \\ Carlos Cardoso Machado ${ }^{2}$
}

\begin{abstract}
RESUMO
O objetivo do presente trabalho é analisar técnica e economicamente operações de extração de madeira com forwarder em função de diferentes volumes por árvore e comprimentos de toras $(2,8 \mathrm{~m} ; 4,0 \mathrm{~m}$ e $5,5 \mathrm{~m})$. Observou-se que a produtividade da extração aumentou diretamente proporcional ao aumento do volume por árvore e ao aumento do comprimento das toras. Os custos de produção, por sua vez, tiveram um comportamento inverso à produtividade.
\end{abstract}

PALAVRAS-CHAVE:

Extração de madeira; forwarder; volume por árvore; comprimentos de toras.

\begin{abstract}
The objective of the present work is to analyze technical and economical forwarder operations in function of the tree volume and different log length $(2,8 \mathrm{~m} ; 4,0 \mathrm{~m}$ and $5,5 \mathrm{~m})$. It was observed that the forwarder productivity increased directly proportional to the increase of the tree volume and to the log length. The production costs, for your time, had an inverse behavior to the productivity.
\end{abstract}

KEY WORDS:

Forwarder operation; harvesting; volume for tree; log length.

\section{RESUMEN}

El objetivo del presente trabajo es analizar técnica y económicamente operaciones de extracción de madera con "forwarder" en función de diferentes volúmenes por árbol y longitudes de troza. Se observó que la productividad de la extracción aumentó en proporción directa al incremento del volumen por árbol y al incremento de la longitud de troza. Los costos de producción tuvieron un comportamiento inverso a la productividad.

PALABRAS CLAVE:

Extracción de madera, volumen por árbol, longitud de troza. 


\section{INTRODUÇÃO}

$\mathrm{Na}$ atividade florestal, a colheita de madeira é a etapa mais importante do ponto de vista econômico e pode ser descrita por três fases básicas: corte/processamento, extração e transporte.

No Brasil, a modernização das operações florestais, principalmente, aquelas que fazem parte do processo de colheita e transporte de madeira, iniciouse na década de $70 \mathrm{com}$ a produção de maquinário de porte médio e leve para fins florestais pela indústria nacional, como por exemplo, as motosserras e gruas carregadeiras (Salmeron, 1981). A mecanização da colheita de madeira, embora não seja a única forma de racionalização e controle da evolução dos custos, pode proporcionar reduções drásticas em prazos relativamente curtos tendo um lugar de elevada importância nos esforços de aumentar a produtividade e humanização do trabalho florestal (Wadouski, 1998).

\section{OBJETIVO}

Este trabalho tem como objetivo analisar, técnica e economicamente, operações de extração de madeira com forwarder, em plantios de eucalipto, em diferentes volumes por árvore e comprimentos de toras.

\section{METODOLOGIA}

\section{Região de estudo}

A pesquisa foi realizada em áreas da KLABIN BACELL S/A, empresa que produz celulose solúvel Kraft branqueada. Está situada no pólo petroquímico de Camaçari - BA - Brasil. Os povoamentos florestais são de propriedade da COPENER FLORESTAL Ltda.., e estão localizados na região Norte do Estado da Bahia-Brasil. Os dados foram coletados no período de 1996-1999. A empresa utiliza toras de Eucalyptus grandis com diâmetros entre 6 e $40 \mathrm{~cm}$ sem casca. Utiliza o sistema de toras curtas com comprimentos: $2.8 \mathrm{~m} ; 4.0 \mathrm{~m}$ e $5.5 \mathrm{~m}$.

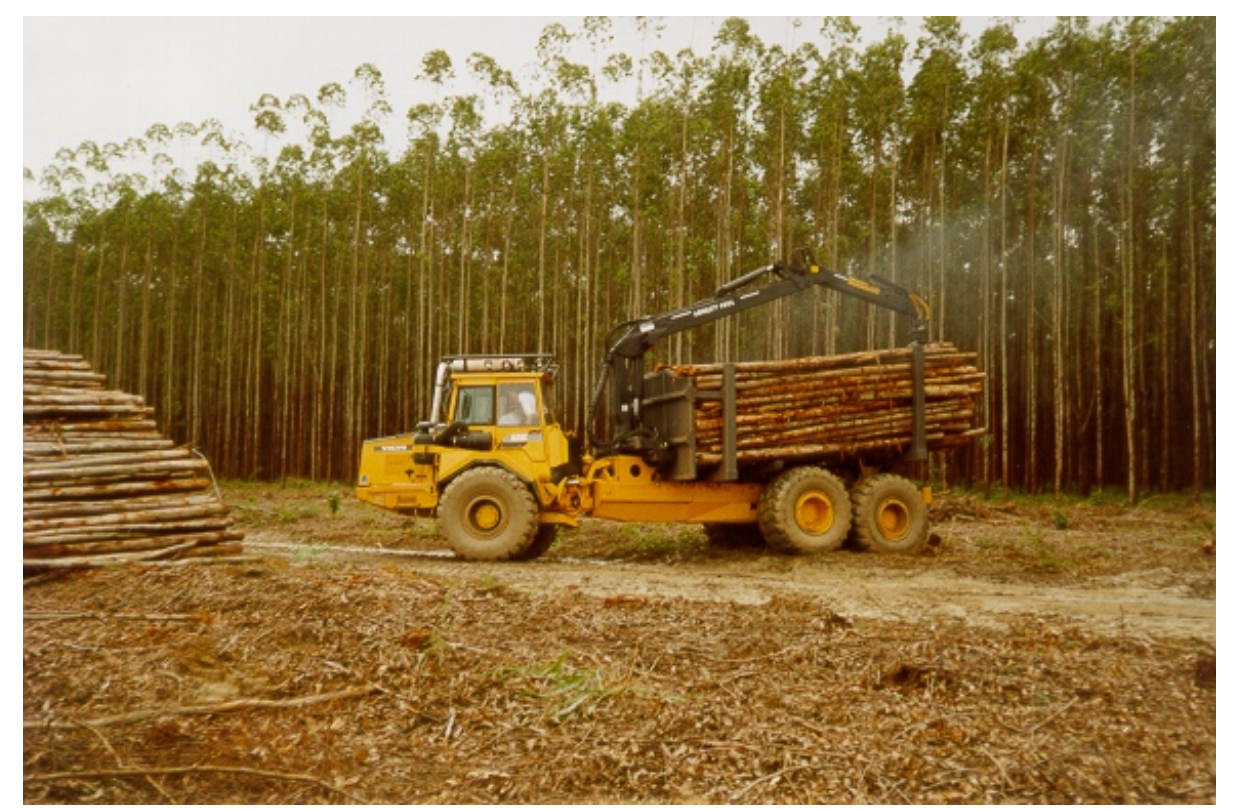

Figura 1. Um Forwarder de tração $6 \times 4$ utilizado na extração de madeira de Eucalyptus grandis 


\section{Produtividade}

Os dados sobre produtividade foram obtidos por meio dos relatórios mensais de produção da empresa. Para obter o volume médio por árvore foram avaliados 14 talhões onde se fez a contagem das árvores e a medição de toda madeira, em metros cúbicos sólidos sem casca. A razão do volume medido pelo número de árvores forneceu o volume médio por árvore em cada talhão. O número total de árvores observadas nesses talhões foi de 537,603 . A razão do volume de madeira extraída pelo número de horas efetivamente trabalhadas forneceu a produtividade média dos mesmos.

\section{Custos Operacionais e de Produção}

Para a determinação dos custos operacionais utilizou-se o método contábil, que usa, simultaneamente, valores estimados e reais. Os custos fixos foram estimados usando-se a metodologia da FAO (1974), citado por Machado e Malinovski (1988). Para os custos variáveis foram utilizados dados reais fornecidos pela empresa.

Custos Fixos. a) Juros. Os juros (J) foram calculados, aplicando-se ao investimento uma taxa de juros, correspondente à que seria, caso o capital fosse proporcionado por alguma agência financeira, da seguinte forma:

Em que:

$$
J=\frac{C a \cdot i \cdot f}{V u}
$$

$$
\begin{aligned}
C a= & \text { custo de aquisição do forwarder } \\
& (\mathrm{R} \$) \\
i= & \text { taxa anual de juros reais }(\%) \\
f= & \text { fator de compensação da forma } \\
& \text { de juros compostos }(0.6) \\
V u= & \text { Vida útil (horas) }
\end{aligned}
$$

b) Depreciação. Segundo Rezende e Valverde (1997), independentemente, do método de depreciação utilizado, a carga anual de depreciação é função do custo de aquisição, da vida útil estimada e do valor residual. $\mathrm{O}$ método de depreciação utilizado foi o linear, onde 0 valor depreciável é obtido ao subtrair do valor de aquisição do bem o seu valor residual. Ao se dividir esse valor pela vida útil estimada, obtém-se a quota de depreciação anual a ser deduzida anualmente:

$$
D=\frac{C a-V r}{V u}
$$

Em que:

$$
\begin{aligned}
& D=\text { Depreciação }(\mathrm{R} \$ / \mathrm{h}) \\
& C a=\text { custo de aquisição da motossera } \\
& \quad(\mathrm{R} \$) \\
& V r=\text { valor de revenda }(\mathrm{R} \$) \\
& V u=\text { Vida útil (horas) }
\end{aligned}
$$

Custos Variáveis. a) Mão-de-Obra (MDO). Os custos de mão-de-obra foram calculados pela razão entre o somatório dos salários mensais de operadores (Sop), ajudantes (Saj) e supervisor da operação (Ssu), acrescidos dos encargos sociais (Es) e equipamentos de proteção individual (EPI) pelo número de horas trabalhadas por mês. Por sua vez, o número de horas trabalhadas por mês é o produto da jornada diária $(\mathrm{Jd})$ pelo número de dias trabalhados (Dt) por mês. A fórmula utilizada para cálculo da mão-deobra é a seguinte:

$M D O=\frac{S o p \cdot E s+S a j \cdot E s+\frac{S s u \cdot E s}{N e}+E P I}{J d \cdot D t}$

b) Peças e Manutenção (PM). Calculou-se esses custos pela razão entre o somatório dos salários mensais da mão-de-obra de oficina (Sof), acrescidos dos encargos sociais, mais as despesas com oficina e ferramentaria (Off), pelo número de horas trabalhadas por mês. A fórmula utilizada foi a seguinte:

$$
P M=\frac{\text { Sof } \cdot E s+O f f}{J d \cdot D t}
$$

c) Combustível e Lubrificantes (CL). Neste cálculo utilizou-se o consumo médio (Cml), em litros por hora, o preço unitário 
do combustível (Pu), o consumo de lubrificantes (Clt) e seu preço unitário(PI).

$$
C L=C m l \cdot P u+C l t \cdot P l
$$

\section{RESULTADOS E DISCUSSÃO}

\section{Estimativa da Produtividade}

No Quadro 1 encontra-se o resumo das informações que serviram de base para a geração dos modelos estatísticos. Nota-se que o tempo médio de carregamento e de descarregamento variou conforme o comprimento da tora. $\mathrm{O}$ maior tempo foi para toras de $2.8 \mathrm{~m}$ de comprimento, com 0.320 e 0.110 hora, respectivamente. Para os comprimentos de 4.0 e $5.5 \mathrm{~m}$, o tempo foi de 0.200 e 0.066 hora, respectivamente. Essa diferença se deve ao fato de que no comprimento de $2.8 \mathrm{~m}$ terem sido colocados dois fardos com toras na carreta, enquanto nos de 4.0 e $5.5 \mathrm{~m}$, apenas um fardo. Os tempos de viagem com e sem carga foram os mesmos para os três comprimentos de toras, ou sejam, $\quad 0.015$ e 0.008 hora, respectivamente. Pode-se observar que o ciclo total de operação e a produtividade do forwarder variaram em função do comprimento da tora e da distância de extração. A produtividade foi sempre maior no maior comprimento de toras, na mesma distância.

Comparando-se a extração de toras de 4.0 e $2.8 \mathrm{~m}$, nota-se que o tempo do ciclo operacional de $4.0 \mathrm{~m}$ foi inferior ao de $2.8 \mathrm{~m}$, todavia as produtividades de ambos foram praticamente iguais para

Quadro 1. Elementos do ciclo operacional e da produtividade do forwarder para diferentes comprimentos de toras e distâncias de extração

\begin{tabular}{|c|c|c|c|c|c|c|c|}
\hline \multirow{2}{*}{$\begin{array}{c}\text { COMP. } \\
\text { DA TORA } \\
(\mathrm{m})\end{array}$} & \multirow{2}{*}{$\begin{array}{c}\text { DIST. } \\
\text { EXTRAÇÃO } \\
(\mathrm{m})\end{array}$} & \multicolumn{4}{|c|}{ ELEMENTOS DO CICLO OPERACIONAL (h) } & \multirow{2}{*}{$\begin{array}{l}\text { CICLO } \\
\text { TOTAL } \\
\text { (h) }\end{array}$} & \multirow{2}{*}{$\begin{array}{c}\text { PRODUTI } \\
\text { VIDADE } \\
\left(\mathrm{m}^{3} / \mathrm{h}\right)\end{array}$} \\
\hline & & Carga & Descarga & $\begin{array}{l}\text { Viagem com } \\
\text { Carga }\end{array}$ & $\begin{array}{c}\text { Viagem sem } \\
\text { Carga }\end{array}$ & & \\
\hline \multirow{6}{*}{2.8} & 50 & 0.320 & 0.110 & 0.015 & 0.008 & 0.453 & 22.08 \\
\hline & 100 & 0.320 & 0.110 & 0.027 & 0.013 & 0.470 & 21.28 \\
\hline & 150 & 0.320 & 0.110 & 0.038 & 0.018 & 0.486 & 20.58 \\
\hline & 200 & 0.320 & 0.110 & 0.050 & 0.023 & 0.503 & 19.88 \\
\hline & 250 & 0.320 & 0.110 & 0.062 & 0.028 & 0.520 & 19.23 \\
\hline & 300 & 0.320 & 0.110 & 0.073 & 0.033 & 0.536 & 18.66 \\
\hline \multirow{6}{*}{4.0} & 50 & 0.200 & 0.066 & 0.015 & 0.008 & 0.289 & 25.16 \\
\hline & 100 & 0.200 & 0.066 & 0.027 & 0.013 & 0.306 & 23.76 \\
\hline & 150 & 0.200 & 0.066 & 0.038 & 0.018 & 0.322 & 22.58 \\
\hline & 200 & 0.200 & 0.066 & 0.050 & 0.023 & 0.339 & 21.45 \\
\hline & 250 & 0.200 & 0.066 & 0.062 & 0.028 & 0.356 & 20.42 \\
\hline & 300 & 0.200 & 0.066 & 0.073 & 0.033 & 0.372 & 19.54 \\
\hline \multirow{6}{*}{5.5} & 50 & 0.200 & 0.066 & 0.015 & 0.008 & 0.289 & 34.60 \\
\hline & 100 & 0.200 & 0.066 & 0.027 & 0.013 & 0.306 & 32.68 \\
\hline & 150 & 0.200 & 0.066 & 0.038 & 0.018 & 0.322 & 31.06 \\
\hline & 200 & 0.200 & 0.066 & 0.050 & 0.023 & 0.339 & 29.50 \\
\hline & 250 & 0.200 & 0.066 & 0.062 & 0.028 & 0.356 & 28.09 \\
\hline & 300 & 0.200 & 0.066 & 0.073 & 0.033 & 0.372 & 26.88 \\
\hline
\end{tabular}


uma mesma distância de extração. Tal fato se deve ao maior volume de carga ter sido transportado com toras de $2.8 \mathrm{~m}$ (10 $\mathrm{m}^{3}$ ), quando comparado com o de $4,0 \mathrm{~m}$ $\left(7.50 \mathrm{~m}^{3}\right)$. Utilizando-se os dados do Quadro 1 obteve-se as equações que estimam a produtividade do forwarder. Foram executados vários testes de ajustes para modelos simples, ou seja, ajustar duas variáveis (distância de extração e produtividade) num conjunto de modelos pré-definidos. $O$ modelo selecionado foi o linear mostrado no Quadro 2 e figura 2. Os dados foram avaliados estatisticamente por meio de funções de regressão tendo como variável dependente a produtividade e como variável independente 0 volume por árvore. Mediante os vários modelos de regressão ajustados, utilizaram-se como critérios de seleção a significância $(F)$ do modelo e o maior coeficiente de determinação ajustado $\left(r^{2}\right)$.

Observou-se que para todas as equações o $\mathrm{F}$ foi significativo em nível de $5 \%$ de probabilidade e os coeficientes de determinação, em todas as situações, está correlacionado positivamente com a variável volume por árvore.

Ao se comparar a extração de madeira nos três comprimentos de toras, observa-se que com $5.5 \mathrm{~m}$, a produtividade é, em média, $23 \%$ superior ao de 4.0 metros e, em média, 52\% superior ao de $2.8 \mathrm{~m}$. A produtividade para as toras de $5.5 \mathrm{~m}$ e um volume por árvore de $0.09 \mathrm{~m}^{3}$, é aproximadamente, igual para as toras de 4.0 metros em um volume por árvore de $0.19 \mathrm{~m}^{3}$.

\section{Determinação dos custos operacionais e de produção}

O Quadro 3 apresenta a composição dos custos operacionais do forwarder.

O Quadro 4 e a figura 3 mostram o comportamento dos custos de produção em função do volume por árvore e comprimento de tora. Observa-se que para um mesmo volume por árvore, o custo de produção para toras de $5.5 \mathrm{~m}$ é $21.89 \%$ inferior ao de toras de $4.0 \mathrm{~m} \mathrm{e}$ $95 \%$ inferior ao de toras de $2.8 \mathrm{~m}$.

Pode-se observar no Quadro 4 e Figura 3 que o custo de produção do Forwarder é inversamente proporcional ao volume por árvore. O custo de produção é mais sensível, ainda, para a situação de toras de 2.8 metros de comprimento. Pode-se observar, também, que para um mesmo volume por árvore, o custo de produção é sempre menor para as toras mais compridas $(5.5 \mathrm{~m})$.

Quadro 2. Equações para estimar a produtividade do forwarder em função da distância de extração para diferentes comprimentos de toras

\begin{tabular}{lccl}
\hline MÁQUINA & $\begin{array}{c}\text { COMPRIMENTO } \\
\text { FLORESTAL }\end{array}$ & EQUAÇÃO & $\mathrm{R}^{2}$ \\
\hline \multirow{2}{*}{ Forwarder } & $2.8 \mathrm{~m}$ & $\mathrm{P}=22.67512-0.01367 \times \mathrm{DE}$ & 0.99 \\
\cline { 2 - 4 } & $4.0 \mathrm{~m}$ & $\mathrm{P}=26.07083-0.0224 \times \mathrm{DE}$ & 0.99 \\
\cline { 2 - 4 } & $5.5 \mathrm{~m}$ & $\mathrm{P}=36.31632-0.03289 \times \mathrm{DE}$ & 0.98 \\
\hline $\mathrm{P}=$ produtividade $\left(\mathrm{m}^{3} / \mathrm{h}\right)$. & & \\
$\mathrm{DE}=$ distância de extração $(\mathrm{m})$. & &
\end{tabular}




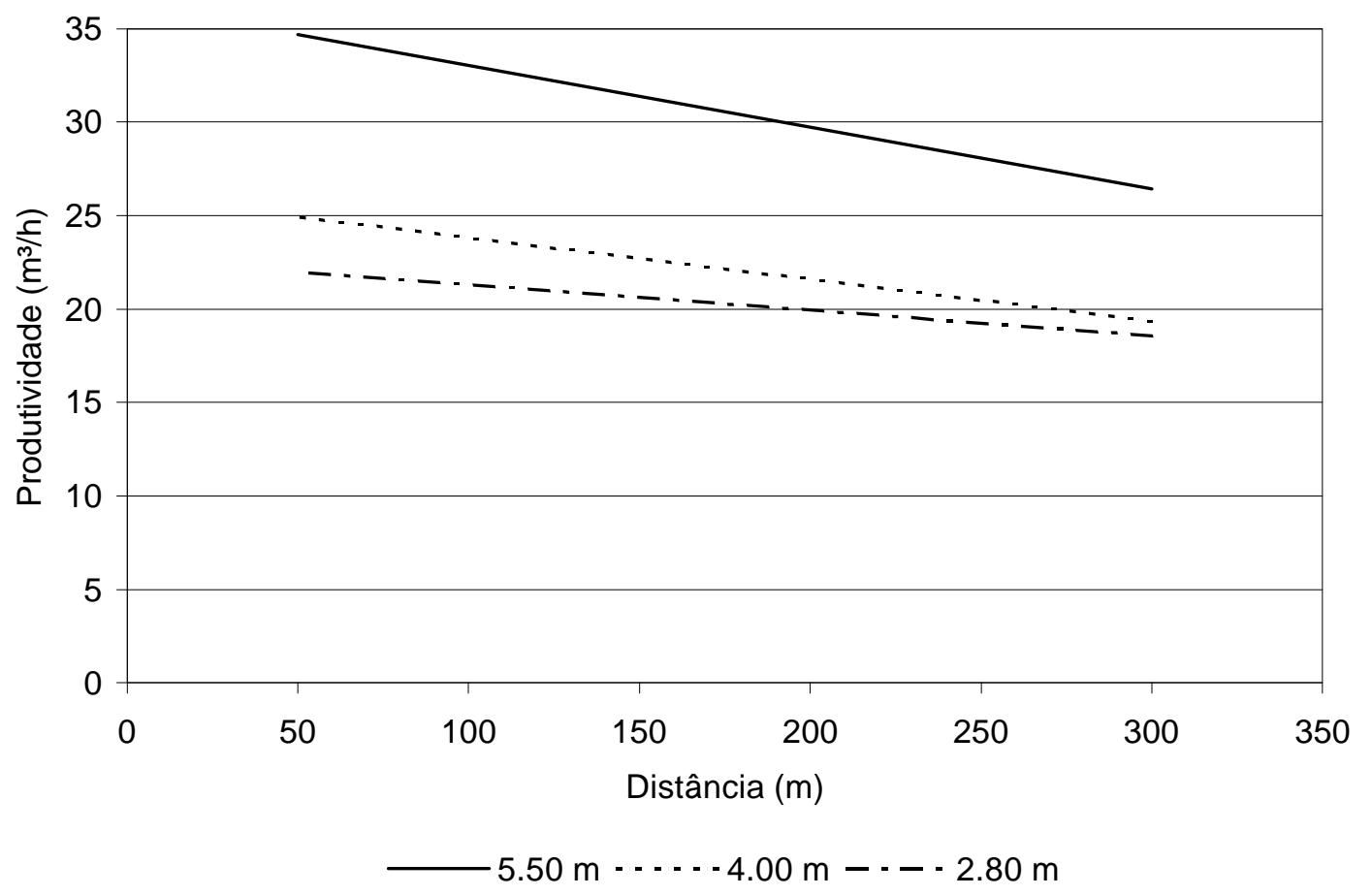

Figura 2. Produtividade do forwarder em função da distancia de extração e comprimento de toras

Quadro 3. Composição dos custos operacionais do forwarder

\begin{tabular}{lc}
\hline ELEMENTOS DE CUSTO & R\$/HORA \\
\hline Juros & 5.29 \\
\hline Depreciação & 18.00 \\
\hline Mão-de-obra & 5.55 \\
\hline Peças/Manut. & 13.94 \\
\hline Comb./Lubr. & 9.60 \\
\hline Total & 52.38 \\
\hline 1 Real $(\mathrm{R} \$)=1.85$ US $\$$
\end{tabular}


Quadro 4. Custos de produção do Forwarder $\left(\mathrm{R} \$ / \mathrm{m}^{3}\right)$ em função do volume por árvore para diferentes comprimentos de toras

\begin{tabular}{cccc}
\hline $\begin{array}{c}\text { VOLUME POR } \\
\text { ÁRVORE } \\
\left(\mathrm{m}^{3}\right)\end{array}$ & \multicolumn{3}{c}{ COMPRIMENTO DE TORAS } \\
\cline { 2 - 4 }$(\mathrm{m})$
\end{tabular}

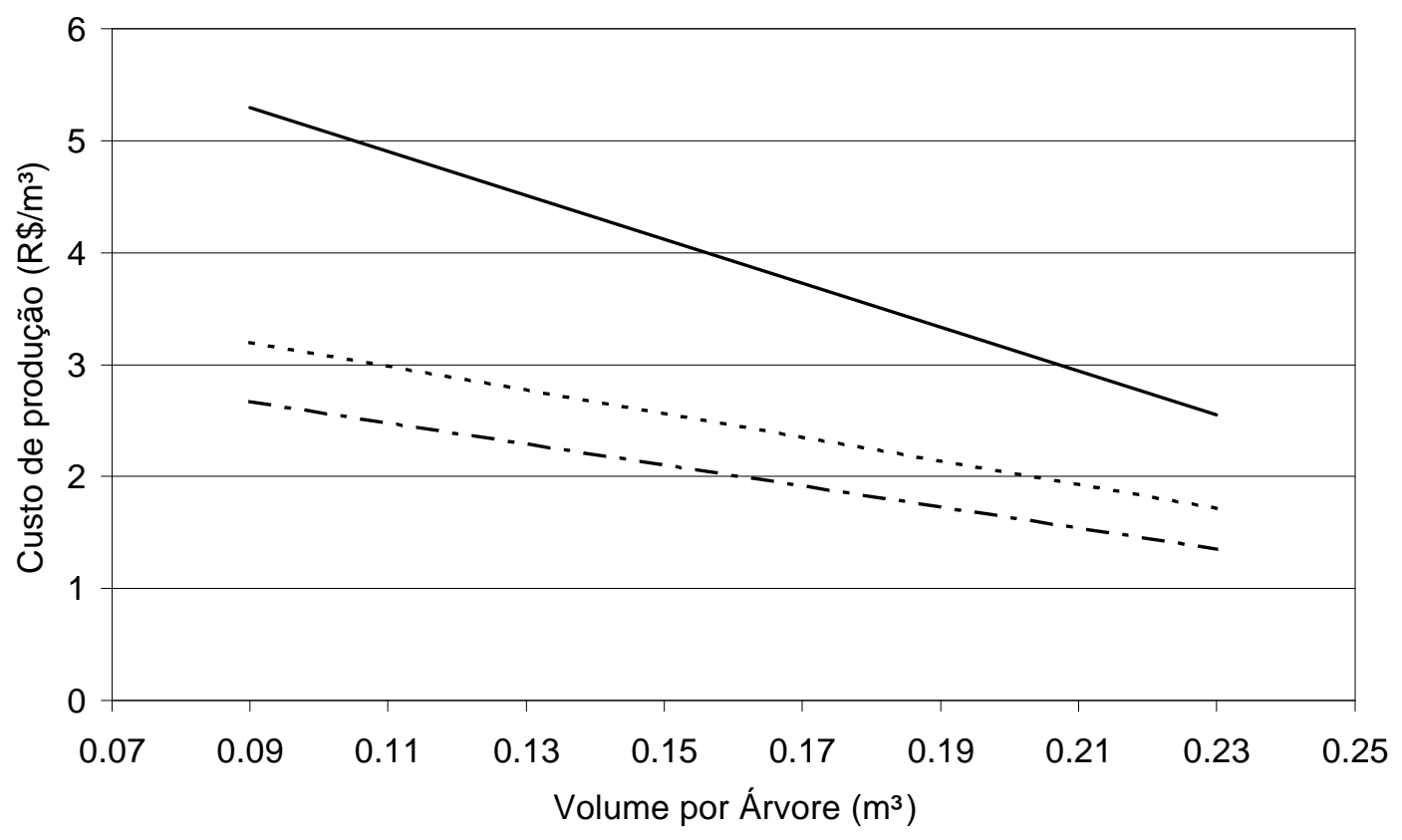

$-2.8 \mathrm{~m} \cdots 4.0 \mathrm{~m}---5.5 \mathrm{~m}$

Figura 3. Custo de produção do forwarder em função do volume por árvore e comprimentos de toras 


\section{CONCLUSÕES}

Com base nos resultados pode-se concluir:

a) Os Forwarders operando com toras de $5,5 \mathrm{~m}$ de comprimento são mais produtivos do que nos comprimentos de 4,0 e 2,8 m, na mesma distância de extração;

b) A produtividade do forwarder cresce inversamente proporcional à distân cia de extração em um mesmo comprimento de toras;

c) $\mathrm{O}$ volume por árvore e o comprimento das toras tem uma relação inversa com o custo de produção do Forwarder. Maiores comprimentos de toras e volumes por árvore traduz-se em menores custos de produção.

\section{REFERÊNCIAS}

FAO. 1974. Logging and log transport in man-made forests in developing countries. Forest Paper - FAO 18. Roma. 90p.

Machado, C.C. e Malinovski, J.R. 1988. Ciência do trabalho florestal. Viçosa, MG. UFV. Impr. Univ. 65p.

Machado, C.C. 1989. Exploração florestal, 6. Viçosa, MG, UFV, Imprensa Universitária, 34p.

Rezende, J.L.P. e Valverde, S.R. 1997. Princípios de depreciação de máquinas e equipamentos. Revista Árvore 21(1):99-111.

Salmeron, A. 1981. A mecanização da exploração florestal. Piracicaba: IPEF. Circular técnica 88. p: 1-10

Wadouski, L.H. 1998. Fatores determinantes da produtividade $\mathrm{e}$ dos custos na colheita de madeira. In.: X Seminário de atualização sobre sistemas de colheita de madeira e transporte florestal. UFPr. Curitiba. p: 77-84. $\diamond$

1 KLABIN BACELL S/A. Rua Alfa, 1033-42810-000 Camaçari. Bahia, Brasil. c.e.: slsantos@klabinbacell.com.br

2 Forestry Department. Federal University of Viçosa. 36571000 Viçosa, Brasil. c.e.: machado@mail.ufv.br

Manuscrito recibido el 1 de noviembre de 2000

Aceptado el 6 de febrero de 2001

Este documento se debe citar como:

Martins S., S.L. y C.C. Machado. 2001. Avaliação técnica e econômica da extração de madeira com forwarder em diferentes volumes por árvore e comprimentos de toras. Madera y Bosques 7(2):87-94. 